\title{
Importance of Complete Pathology Reporting for Neuroendocrine Carcinoma: WHO Guidelines Are a Good Start but Not Enough
}

\author{
Wouter T. Zandee ${ }^{\mathrm{a}}$ Jan Maarten van der Zwan ${ }^{\mathrm{b}}$ Wouter W. de Herder ${ }^{\mathrm{a}}$ \\ Marie-Louise F. van Velthuysen ${ }^{c}$ \\ ${ }^{a}$ Department of Internal Medicine, Sector Endocrinology, Rotterdam, The Netherlands; ${ }^{b}$ Department of Research, \\ Netherlands Comprehensive Cancer Organisation (IKNL), Utrecht, The Netherlands; ' Department of Pathology, \\ ENETS Centre of Excellence, Erasmus University Medical Center and Erasmus MC Cancer Institute, Rotterdam, \\ The Netherlands
}

\section{Keywords}

Neuroendocrine carcinoma · Pathology reporting · Quality of care

\begin{abstract}
Background: Neuroendocrine carcinomas (NECS) are diagnosed through a combination of immunohistochemistry (IHC) and morphology according to WHO guidelines. The presence of these crucial components for classification in the pathology report is critical for appropriate understanding of the report especially since terminology and definitions of NEC have been changing a lot lately. Objectives: The aim of this study is to assess the effect of WHO 2010 on the quality of pathology reporting for NEC and to assess the relevance of the criteria demanded. Methods: Patients registered with a NEC (gastrointestinal or unknown origin) in the Netherlands Cancer Registry (NCR) between 2008 and 2012 were included. Local pathology reports were reviewed for reporting of morphology and IHC comparing 2008-2010 (baseline) with 2011-2012. The diagnosis of NEC was confirmed according to WHO 2010, if synaptophysin or chromogranin were positive in a majority of cells and Ki-67 or mitotic count confirmed a grade 3 tumour. Results: 591 patients were registered with a NEC in the NCR. 436 pathology reports were reviewed. $62.2 \%$ of reports described morphol-
\end{abstract}

ogy, IHC and grading in accordance with WHO 2010. Reporting of these parameters increased from 50.0\% in 2008 to $69.2 \%$ in 2012. Large-cell NEC could be confirmed in $45.0 \%$ of patients, increasing from $31.7 \%$ in 2008 to $56.7 \%$ in 2012 ( $p=0.02$ ). Other diagnoses included neuroendocrine tumour (NET) G1/2 13.3\%, small-cell carcinoma 2.8\%, no neuroendocrine neoplasm (NEN) 17.7\%, NEN grade unknown $21.3 \%$. Mean survival was 1.1 years in large cell NEC versus 2.2 years in NET G1/2 ( $p=0.005)$. Conclusion: Implementation of the WHO 2010 guideline is associated with a significant increase in reporting parameters needed for classification. Stratification of patients is more reliable based on reports containing all parameters. Guidelines alone however are not enough to warrant complete reporting; synoptic reports might be needed.

(c) 2020 The Author(s)
Published by S. Karger AG, Basel

\section{Introduction}

Histopathology is fundamental for the diagnosis of neuroendocrine neoplasms (NENs). Biomarkers and imaging can certainly provide circumstantial evidence, but a biopsy is needed to confirm the diagnosis and for prognostic stratification [1]. The histopathological diagnosis is mainly based on neuroendocrine immunohistochem-
(C) 2020 The Author(s)

Published by S. Karger AG, Basel

This article is licensed under the Creative Commons AttributionNonCommercial-NoDerivatives 4.0 International License (CC BYNC-ND) (http://www.karger.com/Services/OpenAccessLicense). Usage and distribution for commercial purposes as well as any distribution of modified material requires written permission.
Wouter T. Zandee, MD

Erasmus Medical Center and Erasmus MC Cancer Institute

Department of Internal Medicine, Sector Endocrinology, Dr. Molenwaterplein 40 NL-3015 GD Rotterdam (The Netherlands)

w.zandee@erasmusmc.nl 
istry (IHC) (chromogranin A and synaptophysin) followed by grading using the Ki-67 and mitotic index. The correct diagnostic classification is of great importance for several reasons: firstly, for the individual patient as diagnosis and grading is critical for the selection of correct treatment. Secondly, a uniform diagnosis is needed to interpret clinical trials and cohort studies. Due to frequently changing definitions and nomenclature around this topic, it is essential that all necessary parameters for the diagnosis of a NEN are mentioned in the pathology report to ensure a reliable and reproducible diagnosis. It is often assumed that publication of a guideline ensures this much needed correct and uniform diagnosing. In the last decades the classification of NENs has evolved from a classification based on embryological origin [2] via a classification based on morphology (well vs. poorly differentiated) and size [3] to a classification based on proliferative activity [4]. In the most recent WHO classification of 2017 of neuroendocrine tumours (NETs) of the pancreas, high-grade tumours are separated again based on morphology [5]. In 2010 the classification of NENs changed, parting with a system defining a high-grade malignant group based on metastases, invasion and differentiation. Simultaneously, the pathology reports should have evolved along with the evolution of NEN classification. Therefore, we set out to investigate whether the implementation of the WHO 2010 guideline actually changed pathology reporting for non-lung neuroendocrine carcinoma (NEC) in the Netherlands and whether the guideline resulted in uniform and complete pathology reporting. To evaluate the pertinence of complete reporting, the relation with survival was investigated.

\section{Methods}

In the Netherlands, information on all patients with cancer is recorded in the Netherlands Cancer Registry (NCR), which covers 95\% of all cancers. Primary notification occurs through the histopathological diagnosis made by the local pathologist. Demographics, tumour characteristics and treatment are also registered. Morphology and topography of the tumour are recorded using the International Classification of Disease for Oncology, third edition (ICD-O3). The data manager of the NCR is obligated to follow the conclusion of the local pathologist. The data manager selects an ICD-O3 code based on the histopathological conclusion by the pathologist. For this study all patients with a large-cell extrapulmonary NEC (ICD-O3 code M8013) or a NEC not otherwise specified (ICD-O3 code M8246) were included if registered from 2008 to 2012. Only tumours from the gastrointestinal tract or unknown primary tumour were included. From the NCR, vital status, extent of disease, primary origin, age at diagnosis and gender were collected. Using an anonymized link via a third trusted party between
PALGA [6] (the nationwide network and registry of histo- and cytopathology in the Netherlands) and the NCR, pathology reports of these patients were obtained from the time of diagnosis. Microscopy text was reviewed for morphological appearance, IHC, Ki-67 index, mitotic figures, necrosis and differentiation.

As the aim of this study was to demonstrate a change in pathology reporting after the implementation of WHO 2010, we used pathology reports from the period 2008-2010 as baseline to compare with reports after WHO 2010 in the period 2011-2012. This was possible because the ICD-O3 codes did not change in 2010. Percentages of cases with complete reporting of neuroendocrine markers, mitotic index and Ki-67 index were calculated for the period 2008-2010 (baseline) and 2011-2012 to potentially demonstrate differences in diagnostic strategy [7]. Thereafter we aimed to study whether the implementation of the WHO 2010 resulted in a more uniform diagnostic pattern. Using the reports from patients registered in the NCR as well as the information from the original pathology report we classified NECs (M8013 or M8246) using the flow chart shown in Figure 1. We aimed to reproduce the diagnosis of a NEC with use of the described morphology, IHC and grading. First only patients concluded to have a NEC by the local pathologist were included. Pathology conclusions diagnosing small-cell carcinoma, low-grade NEN or carcinoma with neuroendocrine differentiation were regarded to be registered incorrectly. They were excluded because the quality standard defined for NEC did not apply for these tumours.

If no IHC was described at all, the patient was excluded, as IHC might have been performed but could not be tracked with the method used. Then, reports were classified as reporting a smallcell or large-cell carcinoma, either on the basis of the morphological description mentioning moulding of nuclei or lack of cytoplasm or based on the histopathological conclusion. As small-cell NECs can be (focally/faintly) positive or negative for neuroendocrine IHC, these cases were not analysed for the presence of immunohistochemical parameters.

As a third step, large-cell carcinomas required a report of a majority of tumour cells positive with immunohistochemical staining for either chromogranin or synaptophysin. Immunohistochemical staining for CD56 was also registered but was not used for classification as NEN as it is an adhesion molecule and not a neuroendocrine protein [8]. If a neuroendocrine stain was not described, while other (non-neuroendocrine) markers were described, the neuroendocrine marker was assumed not to be performed. If all neuroendocrine stains were negative or weakly positive, the diagnosis was revised to "Large cell carcinoma not otherwise specified" (LCC NOS).

As a fourth step proliferative indexes, mitosis and Ki-67 index, were evaluated. The Ki-67 index was required to be higher than $20 \%$ or the mitotic count needed to be higher than 20 per 10 highpower fields (HPF) in accordance with ENETS/WHO 2010 grading [8], for the tumours to be classified as NEC. When there was a discrepancy between Ki-67 and mitotic count, the highest grade was used for stratification. Patients with a Ki-67 index smaller than $20 \%$ and mitotic count below 20 per $10 \mathrm{HPF}$ were recorded as a low-grade NET. If no Ki-67 assessment was done and mitotic index not mentioned, NENs were classified as "NEN, unknown grade." In certain cases, the Ki-67 or mitotic count was described subjectively (e.g. high/low or abundant). Cases with high or abundant proliferation parameters were classified as NEC and with low levels as NET. 
Fig. 1. Flow chart of neuroendocrine carcinoma (NEC) diagnosis. ${ }^{1}$ Small-cell carcinoma. ${ }^{2}$ Large-cell carcinoma, not otherwise specified (NOS; negative IHC). ${ }^{3}$ Lowgrade neuroendocrine tumour (NET; positive immunohistochemistry, IHC, Ki$67<21 \%$ or mitotic count $<21$ per $10 \mathrm{HPF}$ ). 4 Large-cell neuroendocrine neoplasm $(\mathrm{NEN})$, not graded (positive IHC, missing Ki-67 and mitotic count). ${ }^{5}$ True NEC (positive IHC and Ki-67 $>20 \%$ or mitotic count $>20$ per $10 \mathrm{HPF})$.

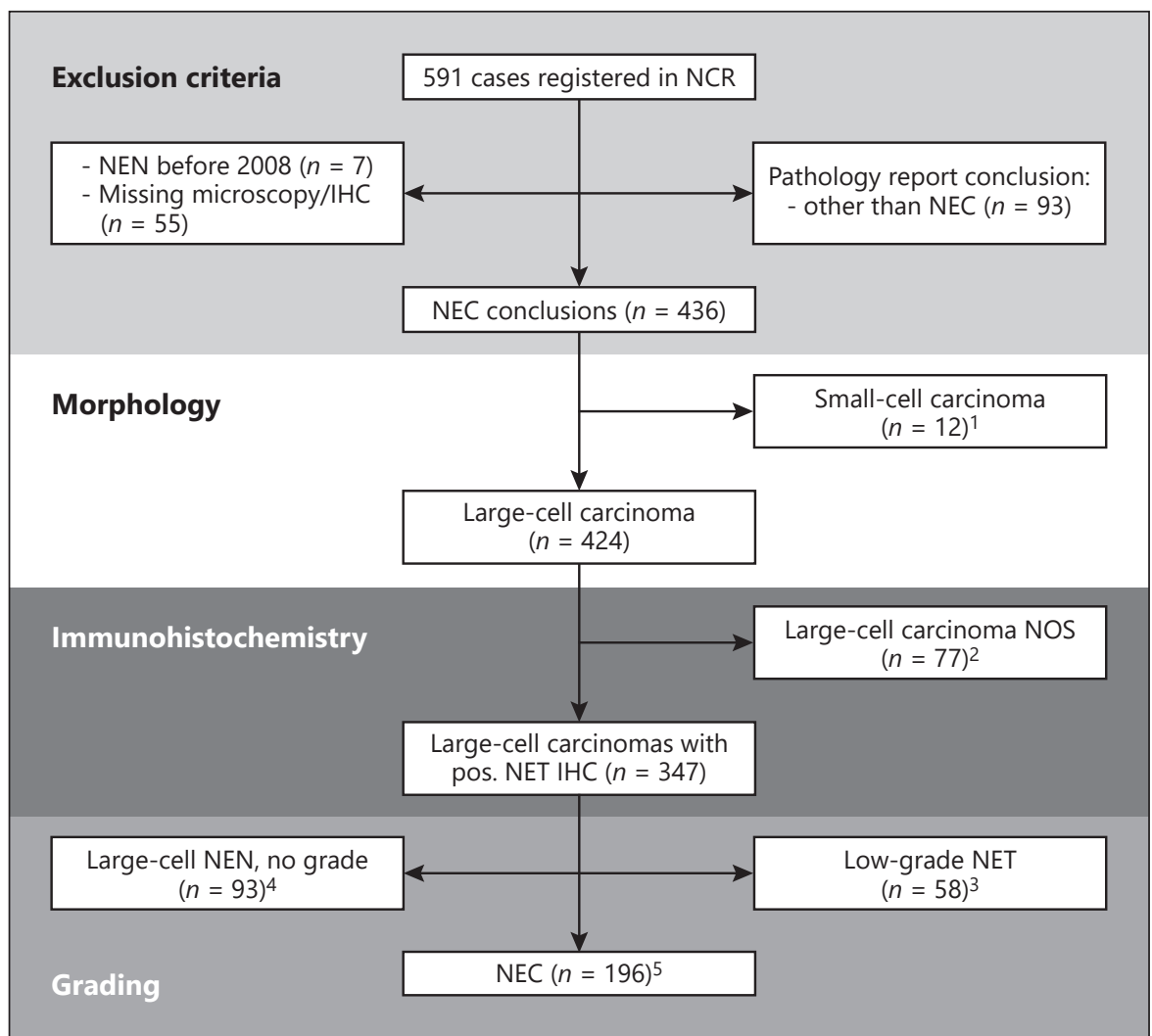

To determine the adherence to the WHO 2010 guideline, percentages of cases with complete reporting of neuroendocrine markers, mitotic index and Ki-67 index were calculated. A $\chi^{2}$ test was performed to test whether the proportion of cases in which all WHO 2010 parameters were reported increased for the years 2008-2012. To validate the model, survival of the different diagnostic groups was estimated with a Kaplan-Meier estimation, and difference in survival was tested with a log-rank test. A univariate analysis was performed to calculate hazard ratios (HRs).

\section{Results}

From 2008 through 2012, a total of 591 patients were registered in the NCR with a large-cell extrapulmonary NEC (M8013) or a NEC not otherwise specified (M8246) from the gastrointestinal tract or unknown origin. Seven patients were excluded because of a NEN being reported in the PALGA pathology registry before 2008, and another 55 patients were excluded due to missing microscopy or IHC and therefore were not applicable for this study. Of the remaining 529 NEC cases in the NCR, 436 (82.4\%) were considered to be a NEC in the local pathology report conclusions. The 93 NCR cases for which the local pathology re- port assigned different conclusions included small-cell carcinoma ( $n=15,2.8 \%)$, carcinoma with neuroendocrine differentiation $(n=29,5.5 \%)$, low-grade NET $(n=42$, $7.9 \%)$ or other carcinomas $(n=7,1.3 \%)$. These tumours were regarded to be misclassified. As the defined quality criteria do not apply for these (non-NEC) tumours, only the 436 patients with a confirmed NEC conclusion in the pathology report were included in the further assessments. These patients were on average 66.8 years old, and $56.2 \%$ were male. Most patients had a primary tumour in the colon, pancreas or of unknown primary origin $(16.3,16.1$ and $41.3 \%$ of cases, respectively; Table 1 ).

\section{Pathology Reporting}

Of all 436 patients it was possible to deduce the cell type from either the histopathological conclusion or the morphological description in the pathology report. Of these 436 patients the morphology of 424 (97.2\%) tumours was described as large cell. The remaining 12 $(2.8 \%)$ small-cell carcinomas were excluded from further analyses.

A synaptophysin stain was reported in 356 patients (84.0\%), and chromogranin staining was reported in 361 
Fig. 2. Completeness of pathology reports of neuroendocrine carcinomas from 2008 to 2012 (percentage). IHC, immunohistochemistry; any neuroendocrine IHC, either synaptophysin or chromogranin was reported. ${ }^{*} p<0.05,{ }^{* *} p<0.001$.

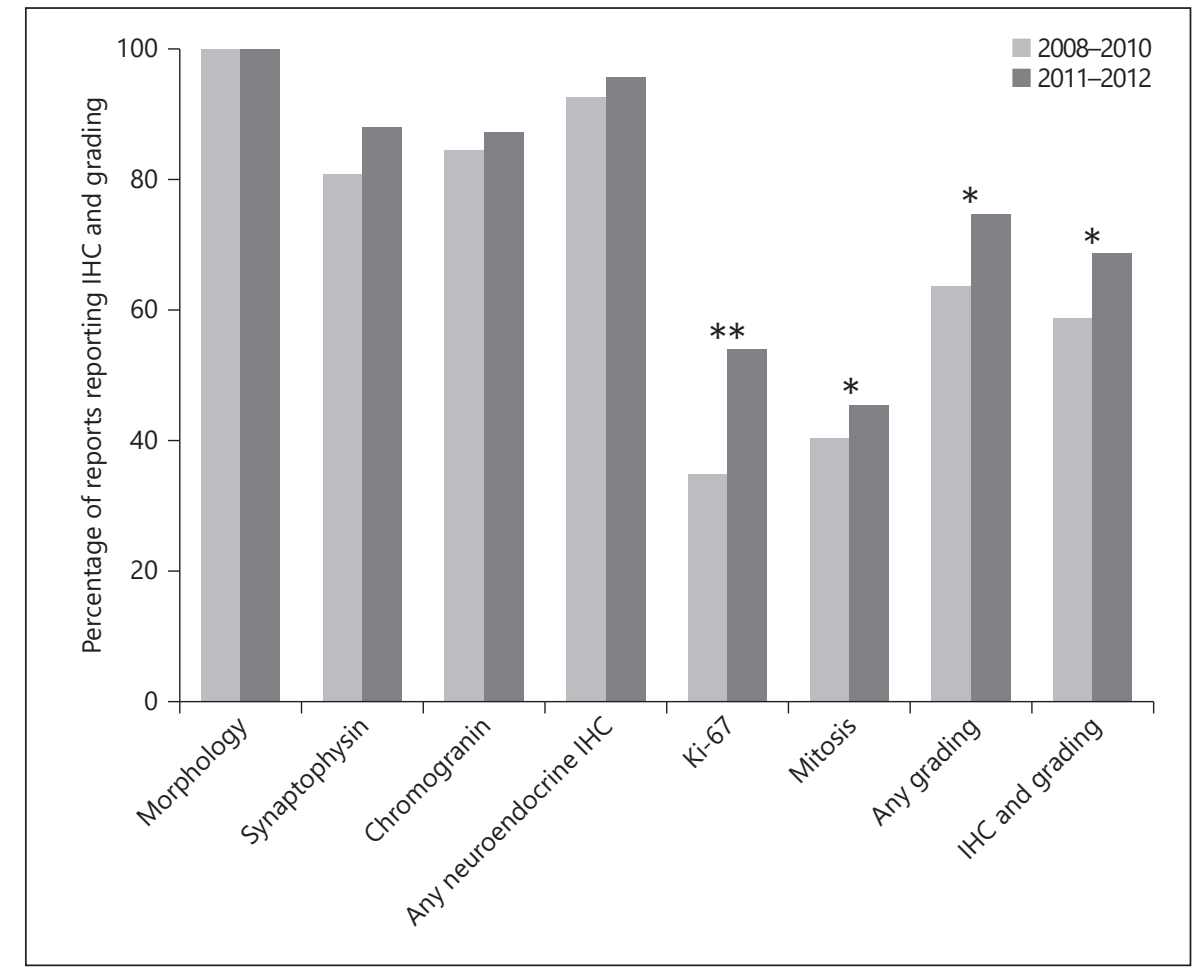

Table 1. Demographic and disease characteristics

\begin{tabular}{lc}
\hline Age, years & $66.7 \pm 12.6$ \\
Male, $n(\%)$ & $245(56.2)$ \\
Primary tumour, $n(\%)$ & \\
$\quad$ Oesophagus & $20(4.6)$ \\
$\quad$ Gastroduodenal & $48(11.0)$ \\
Small intestine & $15(3.4)$ \\
Colon & $71(16.3)$ \\
Rectum & $32(7.3)$ \\
Pancreas & $70(16.1)$ \\
Unknown & $180(41.3)$ \\
Extent of disease, $n(\%)$ & \\
Localized & $89(20.4)$ \\
Advanced & $168(38.5)$ \\
Unknown & $179(41.1)$ \\
\hline
\end{tabular}

Characteristics of 436 cases with a conclusion of neuroendocrine carcinoma in their local pathology reports. Age: mean \pm SD

(85.1\%) patients (Fig. 2). Of 398 (93.9\%) tumours at least one neuroendocrine marker was reported. Grade was reported in smaller amounts of patients: Ki-67 was reported in 185 (43.6\%) patients and mitotic rate in 180 (42.5\%). Sixty-nine percent of cases could be graded because either Ki-67 $(n=107,25.2 \%)$, mitotic rate $(n=112,26.4 \%)$ or

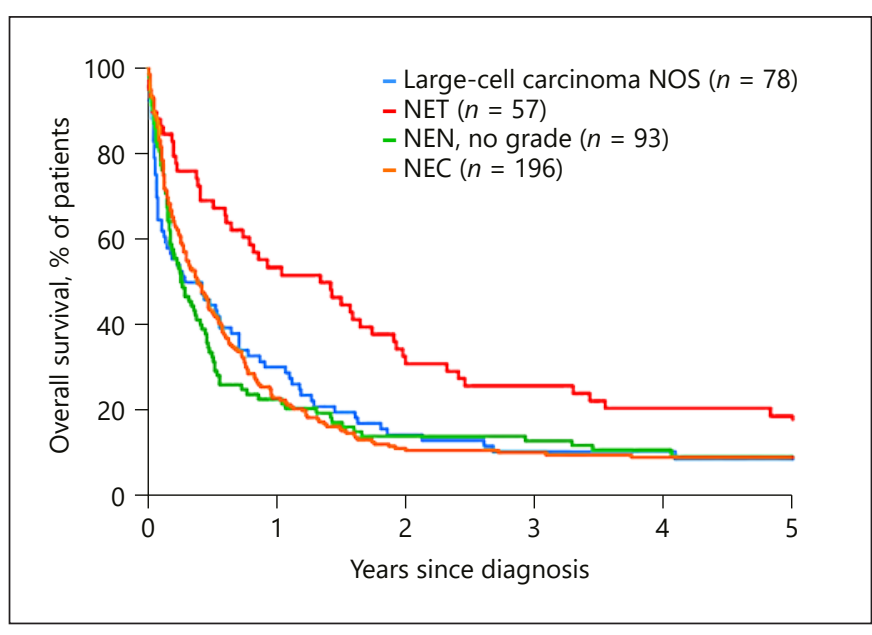

Fig. 3. Overall survival stratified for diagnosis. Results of the Kaplan-Meier analysis of overall survival $(p=0.02)$.

both ( $n=73,17.2 \%)$ were reported. Altogether, in 268 (63.2\%) patients all necessary biomarkers for diagnosis and grading could be assessed. In 2008, 50.0\% of reports included IHC and grading, increasing to $70.6 \%$ in 2012 $(p=0.01)$. This was mainly determined by the reporting of grade (mitosis or Ki-67), increasing from $63.7 \%$ in 
Table 2. Diagnosis per year

\begin{tabular}{|c|c|c|c|c|c|c|}
\hline & 2008 & 2009 & 2010 & 2011 & 2012 & Total \\
\hline Neuroendocrine carcinoma, $n(\%)$ & $19(31.7)$ & $35(42.7)$ & $35(43.2)$ & $48(48.5)$ & $59(57.8)$ & 196 \\
\hline Low-grade neuroendocrine tumour, $n(\%)$ & $10(16.7)$ & $19(23.2)$ & $8(9.9)$ & $12(12.1)$ & $8(7.8)$ & 58 \\
\hline Neuroendocrine neoplasia, no grade, $n(\%)$ & $15(25.0)$ & $18(22.0)$ & $21(25.9)$ & $23(23.2)$ & $16(15.7)$ & 93 \\
\hline Large-cell carcinoma NOS, $n(\%)$ & $16(26.7)$ & $10(12.2)$ & $17(21.0)$ & $16(16.2)$ & $19(18.6)$ & 77 \\
\hline Total & 60 & 82 & 81 & 99 & 102 & 424 \\
\hline
\end{tabular}

Reproducibility of diagnosis with parameters of WHO 2010. NOS, not otherwise specified.

$2008-2010$ to $74.6 \%$ in $2011-2012$ (Fig. $2, p=0.01$ ). The increase in Ki-67 reporting alone was higher: from $34.5 \%$ in 2008-2010 to $53.7 \%$ for $2011-2012$ ( $p<0.001)$. Necrosis and differentiation were seldom reported (25.2 and $24.3 \%)$.

\section{Classification}

Reviewing conclusions and description of morphology in the pathology reports demonstrated that $196(46.2 \%)$ patients were diagnosed with positive neuroendocrine markers and either mitosis or Ki-67 compatible with a NEC in accordance with WHO 2010 guidelines (Fig. 1). Other diagnoses included low-grade NETs $(n=57$, $13.4 \%)$, and $78(18.4 \%)$ had a large-cell carcinoma but the neuroendocrine differentiation could not be confirmed with IHC (LCC NOS), because IHC was negative $(n=23)$; IHC was only described as weakly positive $(n=29)$, or no neuroendocrine markers were described at all $(n=26)$. However, CD56 was positive in $64.1 \%$ of the LCC NOS (50/78), possibly explaining why these tumours were (incorrectly) classified as neuroendocrine. In 93 patients with a NEN (21.3\%), grading was not possible due to missing Ki-67 or mitotic count.

Introduction of the WHO 2010 resulted in a clear increase in reproducibility of NEC diagnoses. From 2008 to $2010,31.7-40.7 \%$ of patients could be classified as NEC with positive IHC and grading. This increased to 48.0 and $56.7 \%$ in 2011 and $2012(p=0.02$, Table 2).

\section{Overall Survival and Prognostic Factors}

Overall survival patterns are in accordance with the histopathological classifications (Fig. 3). Low-grade NETs were associated with the longest survival (mean survival 2.2 years) while the mean survival of NEC was 1.2 years $(p=0.02)$. Mean Ki-67 in the group with low-grade NET was $11 \%$ with more than $70 \%$ of patients having a Ki-67 of $>10$.
Only low-grade NET was a significant predictor in a univariate analysis with an HR of 0.60 (95\% CI: 0.44 0.82). LCC NOS (HR 1.01, 95\% CI: 0.77-1.33) and NEN, no grade (HR 1.06; 95\% CI: 0.82-1.38), showed similar overall survival when compared to NEC.

\section{Discussion}

Diagnostic standards for NEN are described in the WHO 2010 and 2017 guideline for the classification of tumours and the Standard of Care for pathology by ENETS $[5,9]$. The main changes in the WHO 2017 guideline for NETs of the pancreas, which will probably also be adopted for NETs of the gastrointestinal tract, are the slight change in cut-off between grade 1 and 2 NET and the introduction of a further stratification in high-grade NEN. This study demonstrates that implementation of new guidelines for reporting is an effective measure. Implementation of the WHO 2010 was associated with an increase in the use of grading based on Ki-67 or mitotic count from 63.7 to $74.6 \%$, but still many important parameters are lacking in the pathology reports. Even after 2010 , only $43.2-57.8 \%$ of NECs were classified with reporting of all the necessary parameters. The importance of the completeness of the report is highlighted by the fact that due to this completeness, well-differentiated NETs, having a different survival, could be recognized.

Incorrect classification in the NCR seemed to be present. In 93 (17.5\%) patients the conclusion in the pathology report stated a different diagnosis than NEC. Discrepancies in the written information in the patient files apart from pathology reports are a challenge for NCR data managers to report these rare types of cancers correctly. Additionally, due to this diversity it is difficult to build on expertise for the 168 NCR data managers as NEN can be diagnosed in all Dutch hospitals. 
Secondly, a large number of pathology reports did not describe all necessary parameters to diagnose a NEC. This has also been observed in several other cancers. Completeness of pathology reports varies between 10 and $100 \%$ but is often around $30 \%$ [10]. For example, in a recent Italian study, pathology reports for cutaneous melanoma were complete in $77.8 \%$ of cases [11]. Thyroid cancer pathology reports were complete in an Australian study in only $36.4 \%$ of cases [12]. In that perspective, adherence to pathology guidelines for NEN seems comparable with other cancers, and as such the result of the current study is not unique for NEN. The low adherence to pathology guidelines for diagnosis is probably not only caused by the rarity of NENs.

As the diagnosis of a NEC is based on morphology, neuroendocrine IHC and thereafter the demonstration of a high proliferation rate using the Ki-67 index. These parameters have major implications for the treatment of NEN patients. This is demonstrated by the different therapies in ENETS guidelines for high-grade NEC and low-grade NET $[8,13]$. It is therefore essential that the pathologist reports the morphology, IHC and grading in a uniform fashion and that the treating physician can recognize these parameters to verify the diagnosis of the patient. The pathology reports in this study were all written as a narrative, but this way of reporting is famed for missing parameters and thus misinterpretation [14]. Since 2010, in the Netherlands, synoptic reporting is available and widely used since 2013 . These synoptic reports contain standardized reporting language and mandatory parameters. This style of reporting has been shown to significantly increase completeness of pathology reports to nearly $100 \%$ for various cancers $[10,15]$. The next Standard of Care could suggest such a standardized report.

A third reason for the incorrect classification could lie in the previous classification (WHO 2000). This classification defined three groups, based on size, metastases and differentiation. All metastatic disease was classified as endocrine carcinoma with a further differentiation between low- and high-grade malignant behaviour based on differentiation. This could partly explain the pathology conclusions of NEC before 2010, while with the current WHO guideline a low-grade NET would be diagnosed. However, the mandatory differentiation (poor vs. well) was only reported in $28.4 \%$ of reports before 2010 , further underlying the need for a standardized report.

The importance of correct diagnosing is illustrated by the clear difference in survival between low-grade NET and true NEC (Fig. 3). While tumours were considered to be a NEC by the local pathologist, we recognized a NET grade 1 or 2 in $13.7 \%$ of patients due to the reported proliferation markers in the report. These NETs had a significantly longer survival confirming the heterogeneity in the cohort and the importance of uniform reporting and classification. The current study showed that despite implementation of the WHO 2010 guideline, one or more items were missing for classification and grading in $26.8 \%$ of the pathology reports.

Although well-differentiated NEN, especially in resection specimens, can be readily diagnosed without IHC as also stated in the paper of the Delphic consensus process [16], poorly differentiated NECs lose their neuroendocrine morphology and can often only be recognized with neuroendocrine markers. Therefore, the reporting of neuroendocrine markers is essential in this group of patients. Moreover, as these tumours are morphologically seen in a biological continuum, estimation of the proliferative activity by mitotic count and Ki-67 staining is again shown to predict survival. Thus, although publication of the WHO 2010 guideline is associated with a significant increase in reporting parameters needed for classification, essential parameters are still lacking in almost one quarter of reports. Universal application will remain a utopia in narrative reports. Synoptic reporting might give an important boost to meet requirements more quickly.

\section{Statement of Ethics}

Medical ethics committee approval was not required in accordance with the Dutch Medical Research Involving Human Subjects Act.

\section{Disclosure Statement}

The authors have no conflicts of interest to declare.

\section{Author Contributions}

All authors contributed to study design and manuscript writing. W.T.Z., J.M.Z. and M.F.V. contributed to the data collection. All authors approved the final version of the paper. 


\section{References}

1 Hofland J, Zandee WT, de Herder WW. Role of biomarker tests for diagnosis of neuroendocrine tumours. Nat Rev Endocrinol. 2018 Nov;14(11):656-69.

2 Williams ED, Sandler M. The classification of carcinoid tum ours. Lancet. 1963 Feb;1(7275): 238-9.

3 Solcia E. Klöppel Gn, Sobin LH: Histological typing of endocrine tumours. New York: Springer; 2000. https://doi.org/10.1007/9783-642-59655-1.

4 Bosman FT. World Health O: WHO classification of tumours of the digestive system. Lyon, France: IARC Press; 2010.

5 Lloyd RV, Osamura RY, Klöppel G, Rosai J, Bosman FT, Jaffe ES, Lakhani SR, Ohgaki H; World Health Organization; International Agency for Research on Cancer. WHO classification of tumours of endocrine organs, 4th ed. Geneva: WHO Press; 2017.

6 Casparie M, Tiebosch AT, Burger G, Blauwgeers $\mathrm{H}$, van de Pol A, van Krieken JH, Meijer GA. Pathology databanking and biobanking in the Netherlands, a central role for PALGA, the nationwide histopathology and cytopathology data network and archive. Cell Oncol. 2007;29:19-24.

7 Korse CM, Taal BG, van Velthuysen ML, Visser O. Incidence and survival of neuroendocrine tumours in the Netherlands accord- ing to histological grade: experience of two decades of cancer registry. Eur J Cancer. 2013 May;49(8):1975-83.

8 Garcia-Carbonero R, Sorbye H, Baudin E, Raymond E, Wiedenmann B, Niederle B, et al.; Vienna Consensus Conference participants. ENETS consensus guidelines for high-grade gastroenteropancreatic neuroendocrine tumors and neuroendocrine carcinomas. Neuroendocrinology. 2016;103(2):186-94.

9 Perren A, Couvelard A, Scoazec JY, Costa F, Borbath I, Delle Fave G, et al. Antibes Consensus Conference: ENETS consensus guidelines for the standards of care in neuroendocrine tumors: pathology, diagnosis and prognostic stratification. Neuroendocrinology. 2017;105(3):196-200.

10 Sluijter CE, van Lonkhuijzen LR, van Slooten HJ, Nagtegaal ID, Overbeek LI. The effects of implementing synoptic pathology reporting in cancer diagnosis: a systematic review. Virchows Arch. 2016 Jun;468(6):639-49.

11 Tumino R, Minicozzi P, Frasca G, Allemani C, Crocetti E, Ferretti S, et al. Populationbased method for investigating adherence to international recommendations for pathology reporting of primary cutaneous melanoma: results of a EUROCARE-5 high resolution study. Cancer Epidemiol. 2015 Jun;39(3): 424-9.
12 Kahn C, Simonella L, Sywak M, Boyages S, Ung O, O'Connell D. Postsurgical pathology reporting of thyroid cancer in New South Wales, Australia. Thyroid. 2012 Jun;22(6): 604-10.

13 Pavel M, O'Toole D, Costa F, Capdevila J, Gross D, Kianmanesh R, et al.; Vienna Consensus Conference participants. ENETS Consensus Guidelines Update for the Management of Distant Metastatic Disease of Intestinal, Pancreatic, Bronchial Neuroendocrine Neoplasms (NEN) and NEN of Unknown Primary Site. Neuroendocrinology. 2016; 103(2):172-85.

14 Powsner SM, Costa J, Homer RJ. Clinicians are from Mars and pathologists are from Venus. Arch Pathol Lab Med. 2000 Jul;124(7): 1040-6.

15 Srigley JR, McGowan T, Maclean A, Raby M, Ross J, Kramer S, et al. Standardized synoptic cancer pathology reporting: a populationbased approach. J Surg Oncol. 2009 Jun;99(8): $517-24$.

16 Klimstra DS, Modlin IR, Adsay NV, Chetty R, Deshpande V, Gönen M, et al. Pathology reporting of neuroendocrine tumors: application of the Delphic consensus process to the development of a minimum pathology data set. Am J Surg Pathol. 2010 Mar;34(3):30013. 